\title{
Solamargine inhibits gastric cancer progression by regulating the expression of IncNEAT1_2 via the MAPK signaling pathway
}

\author{
RUNJIA FU ${ }^{1}$, XIAOHONG WANG ${ }^{2}$, YING HU $^{2}$, HONG DU $^{1}$, BIN DONG $^{3}$, SHENG AO $^{4}$, \\ LI ZHANG $^{3}$, ZHIJIAN SUN ${ }^{5}$, LIANHAI ZHANG ${ }^{1,2},{\text { GUOQING } \text { LV }^{4} \text { and JIAFU JI }}^{1,2}$ \\ ${ }^{1}$ Department of Surgery, Key Laboratory of Carcinogenesis and Translational Research \\ (Ministry of Education); Departments of ${ }^{2}$ Central Biobank and ${ }^{3}$ Pathology, Peking University Cancer Hospital \\ and Institute, Beijing 100142; ${ }^{4}$ Department of Gastrointestinal Surgery, Peking University Shenzhen Hospital, \\ Shenzhen, Guangdong 518036; ${ }^{5}$ K2 Oncology Co., Ltd., Beijing 100061, P.R. China
}

Received April 5, 2018; Accepted September 6, 2018

DOI: $10.3892 /$ ijo.2019.4744

\begin{abstract}
Solamargine, a derivative from the steroidal solasodine in Solanum species, has exhibited anticancer activities in numerous types of cancer; however, its role in gastric cancer (GC) remains unknown. In the present study, it was demonstrated that Solamargine suppressed the viability of five gastric cancer cell lines in a dose-dependent manner and induced notable alterations in morphology. Treatment with Solamargine promoted cell apoptosis $(\mathrm{P}<0.01)$. Solamargine increased the expression of long noncoding RNA (lnc) p53 induced transcript and lnc nuclear paraspeckle assembly transcript 1 (NEAT1)_2 $(\mathrm{P}<0.01)$ in $\mathrm{GC}$ by reducing the phosphorylation of extracellular signal-regulated kinase (Erk)1/2 mitogen-activated protein kinase (MAPK). To gain insight into the potential mechanism, an Erk1/2 inhibitor (U0126) was applied. The results revealed that lncNEAT1_2 expression levels increased, which was consistent with the effects of Solamargine. Downregulation of IncNEAT1_2 in GC cells revealed no effect on the expression levels of total Erk1/2 and, and counteracted the effect of Solamargine. Solamargine was observed to increase the expression of lncNEAT1_2 via the Erk1/2 MAPK signaling pathway. Of note, the knockdown of lncNEAT1_2 reduced the inhibitory effect of Solamargine $(\mathrm{P}<0.05)$. Additionally, experiments in vivo and in primary GC
\end{abstract}

Correspondence to: Dr Jiafu Ji, Department of Surgery, Key Laboratory of Carcinogenesis and Translational Research (Ministry of Education), Peking University Cancer Hospital and Institute, 52 Fu-Cheng Road, Beijing 100142, P.R. China

E-mail: jijiafu@hsc.pku.edu.cn

Dr Guoqing Lv, Department of Gastrointestinal Surgery, Peking University Shenzhen Hospital, 1120 Lianhua Road, Shenzhen, Guangdong 518036, P.R. China

E-mail: 365973269@qq.com

Key words: Solamargine, long noncoding p53 induced transcript RNA, long noncoding nuclear paraspeckle assembly transcript 1_2, mitogen-activated protein kinase, gastric cancer cells from patients demonstrated that Solamargine significantly suppressed tumor growth $(\mathrm{P}<0.05)$. In vivo analysis of a xenograft mouse model further supported that Solamargine could induce the apoptosis of cancer cells in tumor tissue as observed by a terminal deoxynucleotidyl transferase-mediated dUTP-biotin nick end labeling and H\&E staining $(\mathrm{P}<0.05)$. Experiments in primary GC cells from patients verified the anti-tumor effect of Solamargine. In summary, the findings of the present study indicated that Solamargine inhibited the progression of GC by regulating lncNeat1_2 via the MAPK pathway.

\section{Introduction}

Gastric cancer (GC) is the most common type of gastrointestinal tumor worldwide (1). Recent research has demonstrated that its mortality rate is the third leading cause of mortality in China, and the incidence of GC remains high (2). Therefore, it is urgent to identify chemotherapeutic agents for the treatment of $\mathrm{GC}$ and to improve treatment strategies worldwide.

Numerous components derived from natural substances can inhibit tumor proliferation (3-5). Investigation into natural substances provides a prospective method to understand the mechanism of tumorigenesis. Solamargine, a natural glycoalkaloid compound present in a traditional herbal medicine called Solanum nigrum L. (6), is reported to possess a variety of bioactivities, including antiviral, antitumor and anti-inflammatory properties (7). Previously, numerous studies have investigated the inhibitory effects of Solamargine on tumorigenesis (8-10). Preliminary studies of its role and its potential mechanisms in lung cancer (8), hepatocellular carcinoma (11), breast (12), prostate (13) and ovarian cancers (14), and various tumor cell lines (15) have been reported; however, the effects of Solamargine on GC remain unknown. It was suggested that the inhibitory effects of Solamargine are dependent on the feedback regulation of extracellular signal-regulated kinase (Erk)1/2 mitogen-activated protein kinase (MAPK) $(16,17)$. Whether the potential mechanism of Solamargine involves MAPK regulation requires further investigation.

Long non-coding RNA (lncRNA) refers to transcripts $>200$ nucleotides and do not possess protein coding functions (6). 
Accumulating research has demonstrated that the accurate regulation of signaling pathways by lncRNA serves a pivotal role in the malignant transformation of cells (18). The study of lncRNA p53 induced transcript (IncPINT) in pancreatic cancer demonstrated that its low expression may be an indicator of poor prognosis (19). Marín-Béjar et al (7) revealed that the expression of lncPINT was low in colorectal cancer and its overexpression served a crucial role in tumor progression. Additionally, lnc nuclear paraspeckle assembly transcript 1 (lncNEAT1) was differentially expressed in a variety of solid tumors (20-22); several studies have reported that lncNEAT1 is associated with the prognosis of tumors $(23,24)$. To gain insight into the potential mechanism of the effects of Solamargine on the regulation of IncRNA, further investigation should be conducted.

The present study aimed to determine whether Solamargine is effective against GC. Additionally, analysis of the underlying mechanisms may provide potential therapeutic targets to improve treatment strategies in GC. Furthermore, the anti-tumor effects of Solamargine demonstrated in primary GC cells from patients may contribute to developments into the treatment of gastric cancer.

\section{Materials and methods}

Drug and chemicals. Solamargine was obtained from MedChem Express USA (Monmouth Junction, NJ, US). The drug was dissolved in dimethyl sulfoxide (DMSO; Sigma-Aldrich; Merck KGaA) and prepared as a $10 \mathrm{mM}$ stock solution, which was stored at $-80^{\circ} \mathrm{C}$ and freshly diluted by cell culture medium to the final concentrations $(0.15625,0.3125$, $0.625,1.25,2.5,5,7.5,10$ and $20 \mu \mathrm{M})$ immediately prior to use. U0126 $(10 \mu \mathrm{M})$ was purchased from Selleck Chemicals (Houston, TX, USA) and freshly diluted by cell culture medium to the final concentrations $(10 \mu \mathrm{M})$. GC cell lines (SGC7901 and BGC823) were treated with Erk1/2 MAPK inhibitor U0126 $(10 \mu \mathrm{M})$ for 0,12 and $24 \mathrm{~h}$, or $7.5 \mu \mathrm{M}$ for $36 \mathrm{~h}$ at $37^{\circ} \mathrm{C}$ and $5 \% \mathrm{CO}_{2}$.

Cell viability assay and drug sensitivity assay. The GC cell lines AGS, BGC823, SGC7901, HGC27 and MGC803 were obtained from Chinese Academy of Medical Science (Beijing, China). BGC823, SGC7901 and HGC27 commonly used in our research center were chosen in the further analysis. Primary GC cells were obtained from four patients ( 3 males and 1 female) with GC who underwent radical gastrectomy at Peking University Beijing Cancer Hospital in 2017 (Beijing, China); the age ranged from 34 to 67 years old. Informed consent was provided by all patients. Cell culture medium was Dulbecco's modified Eagle's medium (Gibco; Thermo Fisher Scientific, Inc., Waltham, MA, US) supplemented with $10 \%$ fetal bovine serum (Gibco; Thermo Fisher Scientific, Inc.) at $37^{\circ} \mathrm{C}$ and $5 \% \mathrm{CO}_{2}$. Cells were cultured in cell culture medium containing Solamargine $(0.15625,0.3125,0.625,1.25,2.5$, $5,7.5,10$ and $20 \mu \mathrm{M}$, for the control, equivalent volume of DMSO) for $88 \mathrm{~h}$ at $37^{\circ} \mathrm{C}$ and $5 \% \mathrm{CO}_{2}$. The IncuCyte ZOOM Live-Cell Analysis system (Essen BioScience, Inc., Ann Arbor, MI, USA) which automatically acquires images of living cells and determines the confluence at appropriate time intervals (every $4 \mathrm{~h}$ ), was employed for cell viability analysis. The viability of cells was monitored by an IncuCyte ${ }^{\circledR}$ Live Cell
Analysis system, where viability was assessed by determining the confluence of cells. The half-maximal inhibitory concentration $\left(\mathrm{IC}_{50}\right)$ was calculated using GraphPad Prism statistical software (version 5.0, GraphPad Software, Inc., La Jolla, CA, USA).

Western blot analysis. Protein was extracted from cultured cells and lysed with radioimmunoprecipitation assay lysis buffer (Beyotime Institute of Biotechnology, Haimen, China), phenylmethanesulfonyl fluoride (Beyotime Institute of Biotechnology) and phosphatase inhibitor cocktail I (MedChemExpress, Monmouth Junction, NJ, USA). The protein concentration was measured via the BCA method (BCA Protein Assay kit, Beyotime Institute of Biotechnology), prior to each lysate being boiled for $4 \mathrm{~min}$. Equal quantities $(20 \mu \mathrm{g})$ of protein were added. Proteins were separated by $12 \%$ SDS-PAGE, and electrophoresed at $120 \mathrm{~V}$ for $2 \mathrm{~h}$. Separated proteins were transferred onto a polyvinylidene difluoride membrane at $250 \mathrm{~V}$ for $2 \mathrm{~h}$ on ice. Following protein transfer, the membranes were blocked with $5 \%$ non-fat dry milk in $1 \mathrm{X}$ Tris-buffered saline with Tween-20 $(50 \mathrm{mmol} / \mathrm{l}$ Tris, pH 7.5; $150 \mathrm{mmol} / 1 \mathrm{NaCl} ; 0.1 \%$ Tween-20) at room temperature for $1 \mathrm{~h}$. The membranes were subsequently incubated with antibodies (rabbit anti-human, polyclonal antibodies, 1:1,000; Cell Signaling Technology, Inc., Danvers, MA, USA) against Erk1/2 MAPK (cat. no. 4695), pErk1/2 MAPK (cat. no. 9101), poly (ADP-ribose) polymerase (PARP; cat. no. 9542), cleaved PARP (cat. no. 5625), caspase-7 (cat. no. 9492), cleaved caspase-7 (cat. no. 8438) and GAPDH (cat. no. 5174) at $4^{\circ} \mathrm{C}$ overnight and then with a secondary polyclonal rabbit antibody (goat anti-rabbit; 1:3,000; cat. no. TA130015; OriGene Technologies, Inc., Beijing, China) for $1 \mathrm{~h}$ at room temperature. Finally, a chemiluminescent agent (Thermo Fisher Scientific, Inc.) was added for detection. ImageJ 1.41 software (National Institutes of Health, Bethesda, MD, USA) was used for densitometric analysis.

Reverse transcription-quantitative polymerase chain reaction (RT-qPCR) analysis. Total RNA was extracted from cultured cells using TRIzol ${ }^{\circledR}$ Reagent (Thermo Fisher Scientific, Inc.), according to the manufacturer's protocols. First-strand cDNA was synthetized by RT using the GoScript ${ }^{\mathrm{TM}}$ Reverse System Kit according to the manufacturer's protocols (Promega Corporation, Madison, WI, USA). Subsequently, the reverse transcribed single-stranded cDNA was amplified by SYBR1 Premix Ex Taq II (Takara Bio, Inc.) on an ABI Prism 7500 HT sequence detection system (Applied Biosystems; Thermo Fisher Scientific, Inc.). Each 20- $\mu$ l PCR reaction mixture contained $1 \mu \mathrm{lcDNA}$ product, $0.8 \mu \mathrm{l}$ specific forward/universal primer mix, and $10 \mu \mathrm{l}$ SYBR-Green 2X Universal PCR Master Mix (cat. no. Q141-02; Vazyme, Piscataway, NJ, USA). The thermocycling conditions for qPCR comprised a pre-amplification step of denaturation followed by annealing and extension, and the specific conditions were as follows: $10 \mathrm{~min}$ at $95^{\circ} \mathrm{C}$, followed by 40 cycles of $95^{\circ} \mathrm{C}$ for $3 \mathrm{sec}, 60^{\circ} \mathrm{C}$ for $30 \mathrm{sec}$, and $95^{\circ} \mathrm{C}$ for $15 \mathrm{sec}$. Each sample was tested in triplicate. The raw data were normalized to the data of $\beta$-actin and are presented as the relative expression of 1ncNEAT1_2 or IncPINT. Data analysis was performed using the $2^{-\Delta \Delta \mathrm{Cq}}$ method (25). The primers employed in the present study were 
as follows: NEAT1_2 forward, 5'-GGCCAGAGCTTTGTT GCTTC-3', reverse, 5'-GGTGCGGGCACTTACTTACT-3', PINT forward, 5'-GAACGAGGCAAGGAGCTAAA-3', reverse, 5'-AGCAAGGCAGAGAAACTCCA-3'; and $\beta$-actin forward, 5'-CGTGACATTAAGGAGAAGCTG-3', and reverse, 5'-CTAGAAGCATTTGCGGTGGAC-3'.

Cell cycle analysis. Cells were cultured in cell culture medium containing $10 \mu \mathrm{M}$ Solamargine, or for the control group, cells were treated with DMSO for 6 or $16 \mathrm{~h}$, at $37^{\circ} \mathrm{C}$ and $5 \% \mathrm{CO}_{2}$. The cells were collected, washed and re-suspended in PBS following trypsinization by $0.25 \%$ trypsin for $2 \mathrm{~min}$ at room temperature. and fixed in $70 \%$ ethanol at $-20^{\circ} \mathrm{C}$ overnight. Subsequently, the samples were washed with PBS and then incubated with propidium iodide (PI) staining buffer (Dojindo Molecular Technologies, Inc ) at room temperature for $15 \mathrm{~min}$ prior to analysis with a flow cytometer (BD Pharmingen; BD Biosciences, San Jose, CA, US) using Modfit LT 4.1 software (Verity Software House, Inc., Topsham, ME, USA).

Annexin V-fluorescein isothiocyanate (FITC)/PI apoptosis assay. Cells were cultured in medium containing Solamargine (for the control, equivalent volume of DMSO) for $48 \mathrm{~h}$, the cells were then collected and washed with PBS (Gibco; Thermo Fisher Scientific, Inc.) at $37^{\circ} \mathrm{C}$. The cells were re-suspended in Annexin V Binding Solution, followed by the addition of Annexin V-FITC and PI solution (Annexin V-FITC Apoptosis Detection Kit, Dojindo Molecular Technologies, Inc.) for $20 \mathrm{~min}$ in the dark at room temperature. Finally, Annexin V Binding solution was added and cells were analyzed with BD Accuri $^{\mathrm{TM}}$ C6 software by flow cytometry (BD Biosciences). Quantification of the flow cytometry results demonstrated the fractions of cells in the early and late stage of apoptosis.

Transient transfection assay. LncNEAT1_2-target small interfering (si)RNA (5'-GUCUGUGUGGAAGGAGGA ATT-3') and nonspecific scrambled siRNA (5'-UUCUCCGAA CGUGUCACGUTT-3') were designed and synthesized by Shanghai GenePharma Co., Ltd. (Shanghai, China). Cells (SGC 7901 and BGC 823) were employed according to manufacturer's protocols; $5 \mu 1$ siRNA or negative control siRNA, and $6 \mu 1$ Lipofectamine ${ }^{\circledR} 2000$ (Invitrogen; Thermo Fisher Scientific, Inc.) were used for transfection. After $6 \mathrm{~h}$, the transfection solution was discarded and cell culture medium containing serum was added; cells were cultured for $24 \mathrm{~h}$ prior to the subsequent experiments. To evaluate the efficiency of transfection, the expression of 1ncNEAT1_2 was investigated by RT-qPCR analysis as aforementioned.

Tumor xenograft. The present study was performed in strict accordance with the recommendations of the Guidelines for the Care and Use of Laboratory Animals of the Peking University Cancer Hospital and Institute (26). Female specific pathogen-free BALB/c nude mice weighing 18-20 g (6-8-weeks-old) were employed and housed under $21 \pm 2{ }^{\circ} \mathrm{C}, 50-60 \%$ humidity with normal air and with a $12 \mathrm{~h}$ light/dark cycle. Mice were provided $5 \mathrm{~g}$ food and $6-8 \mathrm{ml}$ water/mouse/day. A total of 10 experimental mice were randomly divided into two groups (5 per group). BCG 823 is mostly used to establish tumor xenograft for research in our center. Mice of each group were administered a subcutaneous injection of BGC823 GC cells $\left(5 \times 10^{5}\right.$ cells per mouse) into the left hind leg. After 10 days, the experimental group was treated with $10 \mathrm{mg} / \mathrm{kg}$ Solamargine once daily by intragastric administration, while the control group was administered PBS for 8 days corresponding to the time for the tumors in control group to reach about $600-800 \mathrm{~mm}^{3}$. Tumor growth was monitored every 2 days (total 18 days) by measuring the width (b) and length (a) of the tumors with calipers. The tumor volume (V) was calculated by the formula: $\mathrm{V}=1 / 2 \mathrm{a} x \mathrm{~b} 2$. Mice were sacrificed for the collection of tumor samples.

Terminal deoxynucleotidyl transferase-mediated dUTP-biotin nick end labeling (TUNEL) assay. Paraffin-embedded mouse tumor tissues fixed with $10 \%$ formalin solution for $12 \mathrm{~h}$ were cut and mounted on slides ( $4 \mu \mathrm{m}$ section) at room temperature. First, the tissue sections were dewaxed and rehydrated in a descending alcohol series. Subsequently, the slides were treated In Situ Cell Death Detection kit, TMR red (Roche Applied Science, Rotkreuz, Switzerland) according to the manufacturer's protocols. Briefly, the slides were treated with $0.1 \mathrm{M}$ citrate buffer, $\mathrm{pH} 6.0$ with microwave irradiation (750 watts) for $1 \mathrm{~min}$. Then, the slides were immersed in $0.1 \mathrm{M}$ Tris- $\mathrm{HCl}(\mathrm{pH} 7.5$ ) containing 3\% BSA and 20\% normal bovine serum (Gibco; Thermo Fisher Scientific, Inc.) at room temperature for $30 \mathrm{~min}$. In addition, $50 \mu \mathrm{l}$ TUNEL reaction mixture per section was added to cover the slides and incubated for another $60 \mathrm{~min}$ at $>37^{\circ} \mathrm{C}$ in a humidified atmosphere in the dark. Finally, the slides were counterstained for $6 \mathrm{~min}$ with DAPI at room temperature (Guangzhou Ribobio, Co., Ltd., Guangzhou, China). The sections were evaluated under a fluorescence microscope (magnification, x63, Lm780, Zeiss AG, Oberkochen, Germany). Apoptotic cells were quantified by measuring the average cell numbers in five random high-power fields of each of sample for analysis.

Haematoxylin and eosin $(H \& E)$ staining. Paraffin-embedded mouse tumor tissues were fixed in $10 \%$ formalin solution for $12 \mathrm{~h}$ at room temperature were cut and mounted on slides ( $4 \mu \mathrm{m}$ thick). The tissue sections were dewaxed and rehydrated in a descending alcohol series. Subsequently, the slides were stained with hematoxylin stain for $1 \mathrm{~min}$ at room temperature. Subsequently, the samples were washed in water for $10 \mathrm{~min}$ and after differentiation in acid alcohol, the slides were stained with eosin for $1 \mathrm{~min}$ at room temperature. High-quality images were obtained using by Aperio CS2 image capture device (magnification, $x 5$ and 20). Necrotic areas were quantified (Aperio ImageScope software, version 12.1, Aperio Technologies; Leica Microsystems, Inc., Buffalo Grove, IL, USA) by measuring the necrotic area (\%) in three slides of each sample for analysis.

Statistical analysis. Every experiment was repeated at least three times, and the data were expressed as the mean \pm standard deviation. The differences between groups were assessed by a Student's t-test or one-way analysis of variance followed by Tukey's post-hoc test. All statistical analyses were performed using SPSS software (version 22.0, IBM Corp., Armonk, NY, USA) and GraphPad Prism statistical software (version 5.0). Comparisons were conducted with the corresponding controls. 
A

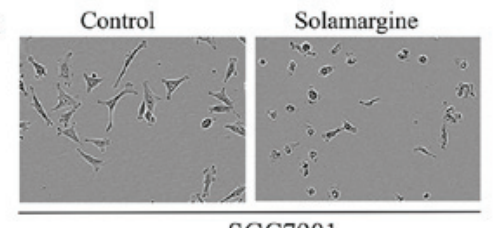

SGC7901

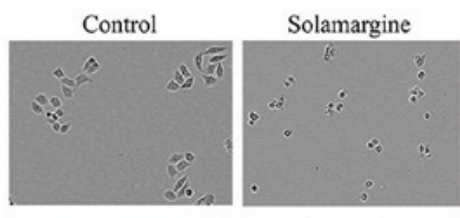

BGC823
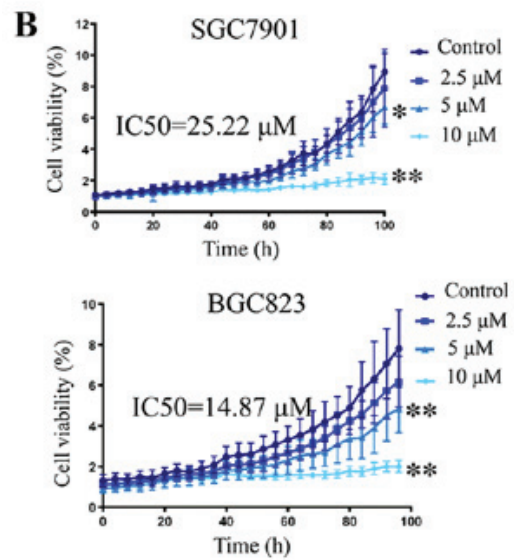

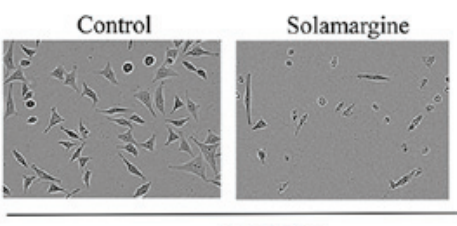

MGC803
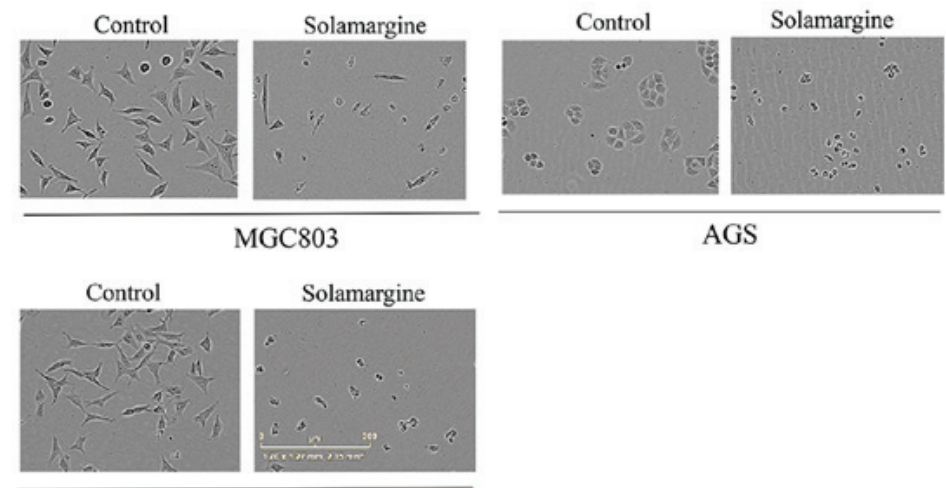

HGC27
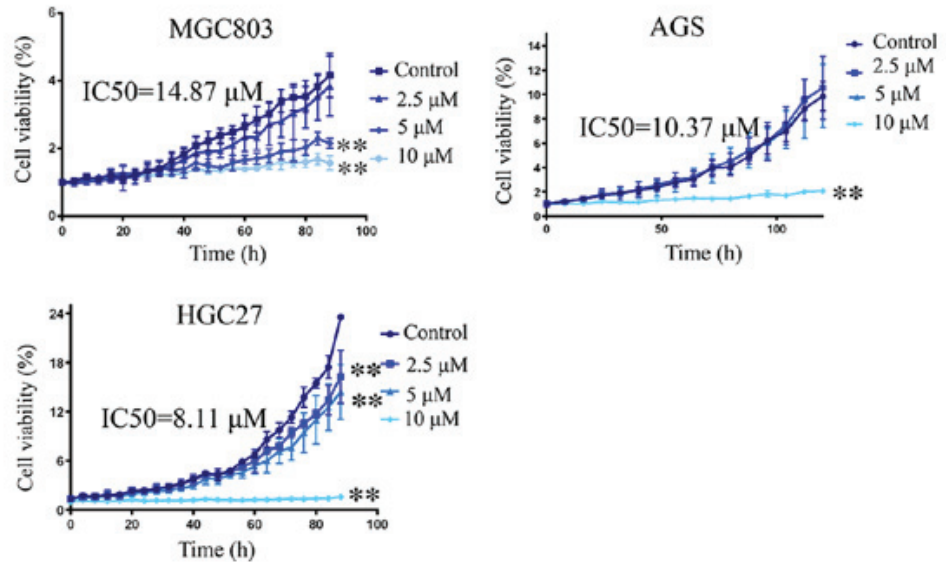

Figure 1. Effects of Solamargine on the morphology and viability of GC cells. (A) Effects of Solamargine on cellular morphology. GC cell lines (AGS, BGC823, SGC7901, HGC27 and MGC803) were treated with Solamargine (10 $\mu \mathrm{M})$ for $48 \mathrm{~h}$. Magnification, x10. (B) Solamargine decreased the viability of GC cells. GC cell lines (AGS, BGC823, SGC7901, HGC27, and MGC803) were treated with different concentrations of Solamargine (0, 2.5, 5 and $10 \mu$ M). Subsequently, the cell viability was determined with the IncuCyte ZOOM Live-Cell Analysis System every $8 \mathrm{~h}$. $\mathrm{P}<0.05$, ${ }^{* *} \mathrm{P}<0.01 \mathrm{vs}$. control. The IC ${ }_{50}$ for $6 \mathrm{GC}$ cell lines were calculated by GraphPad Prism statistical software (version 5.0). GC, gastric cancer; IC $_{50}$, half-maximal inhibitory concentration.

$\mathrm{P}<0.05$ was considered to indicate a statistically significant difference.

\section{Results}

Solamargine suppresses the viability of GC cells and induces alterations in morphology. Notable morphological changes were observed in GC cell lines cultured with Solamargine compared with in the control (Fig. 1A). The volume of cells was reduced, the intercellular junctions were not visible, the nuclei were concentrated; few cells were not firmly adhered and large amounts of cell debris were visible. To verify the effects of Solamargine on viability, the IncuCyte ZOOM Live-Cell Analysis System which can automatically acquire images of living cells and analysis the confluence based on the image to reflect the cell viability was employed. The results of the present study revealed that Solamargine suppressed cell viability in five GC cell lines (AGS, BGC823, SGC7901, HGC27 and MGC803) in a dose-dependent manner and $\mathrm{IC}_{50}$ values are presented in Fig. 1B. Compared with in the control group, the viability of GC cells in the Solamargine group gradually decreased with increasing drug concentrations $(\mathrm{P}<0.05)$.

Solamargine promotes the apoptosis of GC cells. Based on the inhibitory effects and alterations in morphology induced by Solamargine, the apoptotic effect of Solamargine on GC cells was analyzed by Annexin V-FITC/PI double-staining and western blotting. The results demonstrated that the rate of apoptosis in the Solamargine group was significantly higher than that of the control group (Fig. 2). Considering that caspase activation is the key event for apoptosis $(27,28)$, the expression levels of full-length and cleaved caspase proteins were measured by western blotting (Fig. 3A and B). Treatment with Solamargine $(7.5 \mu \mathrm{M}, 12$ or $24 \mathrm{~h})$ significantly enhanced the cleavage of caspase-7 and PARP compared with in the corresponding controls, indicating that Solamargine may promote the apoptosis of GC cells.

Solamargine increases the proportion of GC cells in $G 2 / M$ phase. In addition, the effects of Solamargine on the cell cycle of GC cells was investigated. The results revealed a significant increase in the number of in SGC7901, HGC27 and BGC823 cells in G2/M phase treated with Solamargine $(10 \mu \mathrm{M})$ for $16 \mathrm{~h}$ compared with in the control (Fig. 3C). Additionally, to exclude interference by other factors, alterations resulting from $6 \mathrm{~h}$ treatment with Solamargine in the corresponding GC cell lines were analyzed. The results revealed no significant difference in the number of GC cells exposed to Solamargine and the corresponding control at this time point (Fig. 3D). In summary, the present study reported that Solamargine increased the number of GC cells in $\mathrm{G} 2 / \mathrm{M}$ phase. 


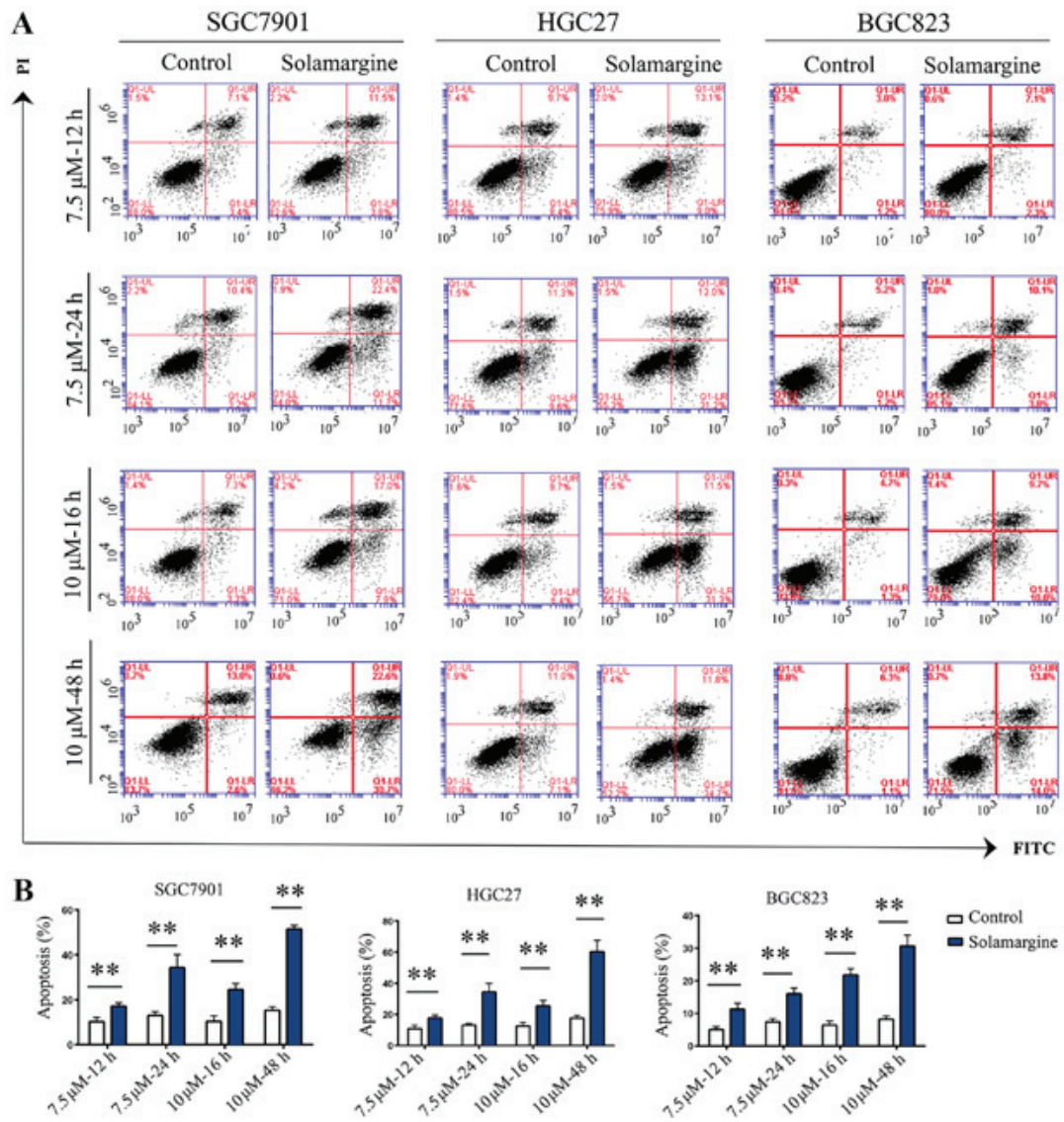

Figure 2. Effects of Solamargine on the apoptosis of GC cells. (A) GC cell lines SGC7901, HGC27 and BGC823 were treated with Solamargine (7.5 or $10 \mu \mathrm{M}$, or DMSO) for 12, 24, 16 or $48 \mathrm{~h}$. (B) Cell apoptosis was analyzed by Annexin V-FITC/PI double-staining and flow cytometry. ${ }^{* * *} \mathrm{P}<0.01$ vs. control. DMSO, dimethyl sulfoxide; FITC, fluorescein isothiocyanate; PI, propidium iodide.
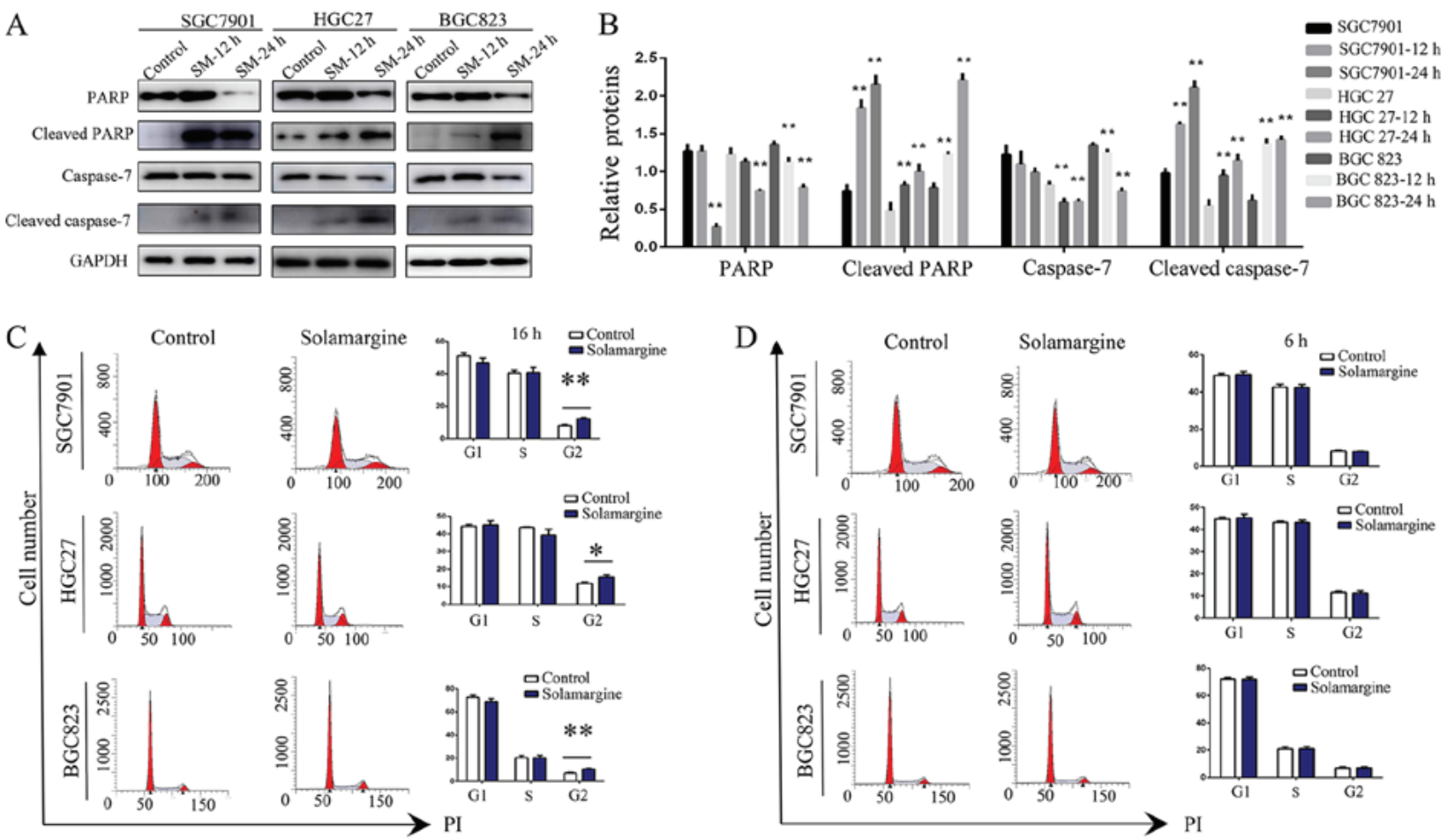

Figure 3. Solamargine enhance the cleavage of caspase-7 and PARP. (A and B) Treatment with Solamargine enhanced the cleavage of caspase-7 and PARP. The GC cell lines SGC7901, BGC823 and HGC27 were treated with Solamargine (7.5 $\mu \mathrm{M})$ for 12 or $24 \mathrm{~h}$. The expression levels of PARP, cleaved PARP, caspase-7 and cleaved caspase-7 were detected by western blotting. (C and D) GC cell lines SGC7901, HGC27 and BGC823 were treated with $10 \mu$ M Solamargine or DMSO for $16 \mathrm{~h}$. The cell cycle was analyzed by PI staining and flow cytometry. ${ }^{*} \mathrm{P}<0.05,{ }^{* *} \mathrm{P}<0.01$ vs. control. GC, gastric cancer; PARP, poly (ADP-ribose) polymerase; PI, propidium iodide. 

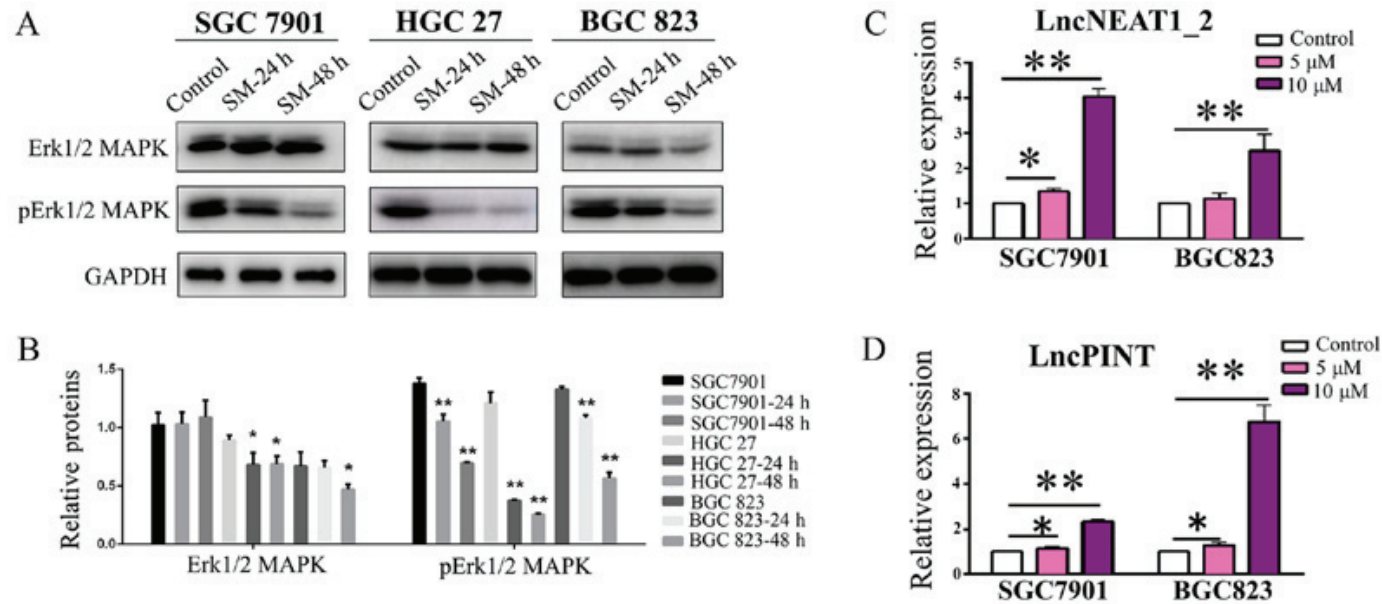

Figure 4. Solamargine inhibits the phosphorylation of Erk1/2 MAPK, and increases the expression of lncPINT and lncNEAT1_2. (A and B) GC cell lines SGC7901, BGC823 and HGC27 were treated with Solamargine $(0$ and $10 \mu \mathrm{M})$ for 24 and $48 \mathrm{~h}$. The expression levels of Erk1/2 MAPK and pErk1/2 MAPK were detected by western blotting. (C and D) Effects of Solamargine $(0,5$ and $10 \mu \mathrm{M})$ on the expression of lncPINT and lncNEAT1_2. ${ }^{*} \mathrm{P}<0.05$, ${ }^{* * *} \mathrm{P}<0.01$ vs. control. Erk1/2, extracellular signal-regulated kinase 1/2; lnc, long noncoding RNA; MAPK, mitogen-activated protein kinase; p, phosphorylated.

A

Erk1/2 MAPK

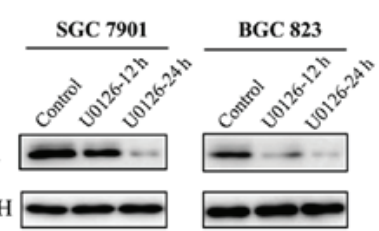

$\mathrm{D}$

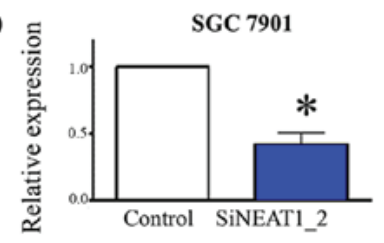

G

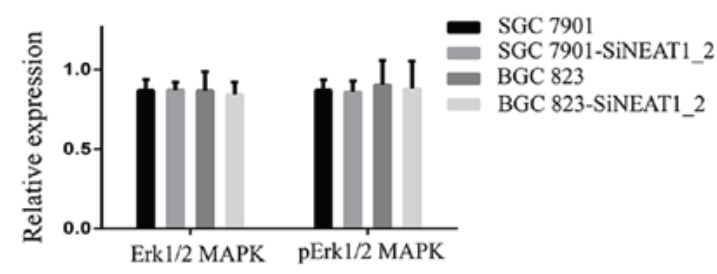

B

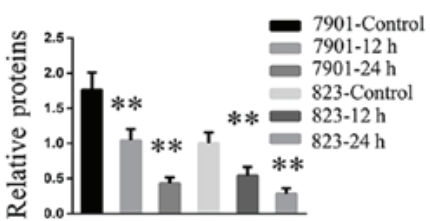

E

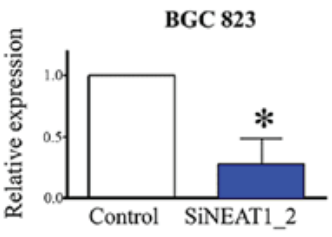

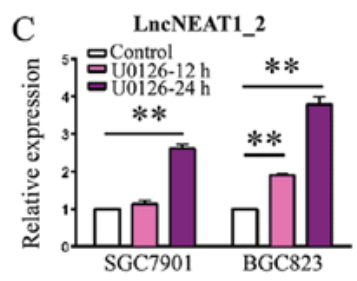

F

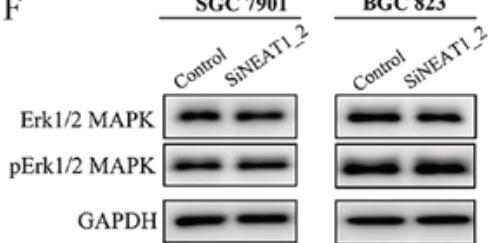

$\mathrm{H}$

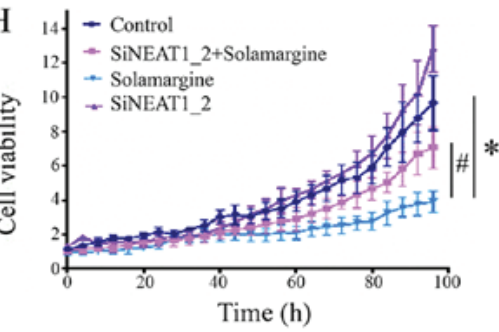

Figure 5. Effects of an Erk1/2 MAPK inhibitor (U0126) on 1ncPINT and lncNEAT1_2 expression and loss-of-function assays. (A and B) Expression of Erk1/2 MAPK. GC cell lines (SGC7901 and BGC823) were treated with Erk1/2 MAPK inhibitor U0126 (10 $\mu \mathrm{M})$ for 0, 12 or $24 \mathrm{~h}$. (C) Inhibition of Erk1/2 MAPK increased the expression of lncNEAT1_2 in SGC7901 and BGC823 . * P $<0.01$ vs. control. (D and E) Knockdown of 1ncNEAT1_2. "P<0.05 vs. control. (F and G) Knockdown of lncNEAT1_2 exhibited no notable effects on the expression of Erk1/2 MAPK or its phosphorylation. (H) Knockdown of lncNEAT1_2 attenuated the inhibitory effects of Solamargine on GC cells. GC cells (SGC7901) were treated with Solamargine (7.5 $\mu \mathrm{M})$ for $36 \mathrm{~h}$. "P<0.05 vs control. ${ }^{\text {"P }}$ < 0.05 vs. Solamargine. SiNEAT1_2, knockdown of lncNEAT1_2; Erk1/2, extracellular signal-regulated kinase 1/2; lnc, long noncoding RNA; MAPK, mitogen-activated protein kinase; $\mathrm{p}$, phosphorylated.

Solamargine inhibits the phosphorylation of Erk1/2 MAPK. The MAPK signaling pathway, an important signaling pathway within eukaryotic cells, serves a key role in the development of tumors and has been reported as a novel target for tumor therapy (17-19). The present study investigated the effects of Solamargine on MAPK activity. As presented in Fig. 4A and B, the expression levels of Erk1/2 and p-Erk1/2 MAPK were significantly reduced in the cell lines in a time-dependent manner following Solamargine treatment, compared with in the corresponding control; however, no significance was observed in the expression levels of Erk1/2 MAPK in SGC7901 cells. These findings indicated that the MAPK signaling pathway is involved in the regulatory effects of Solamargine.

Solamargine increases the expression of IncPINT and IncNEAT1_2. As aforementioned, numerous studies have 
A
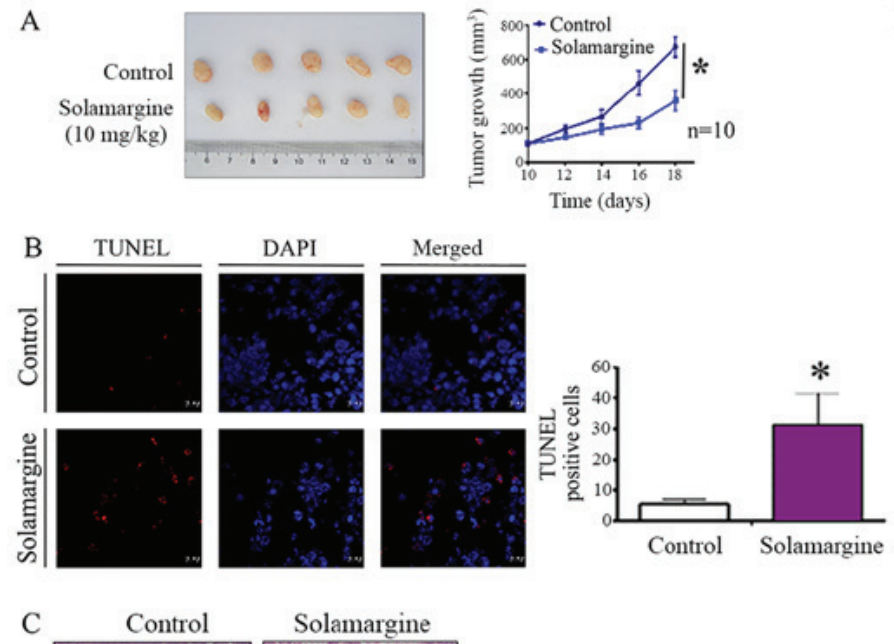

Solamargine
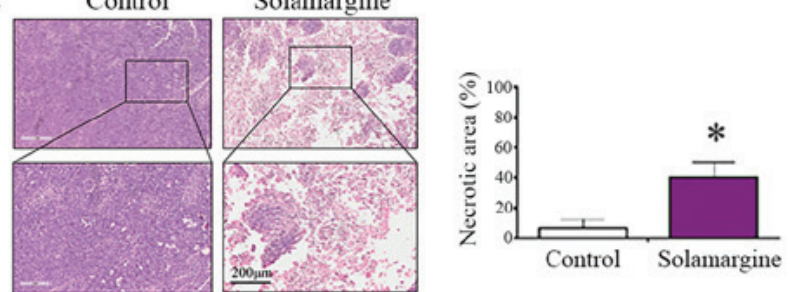
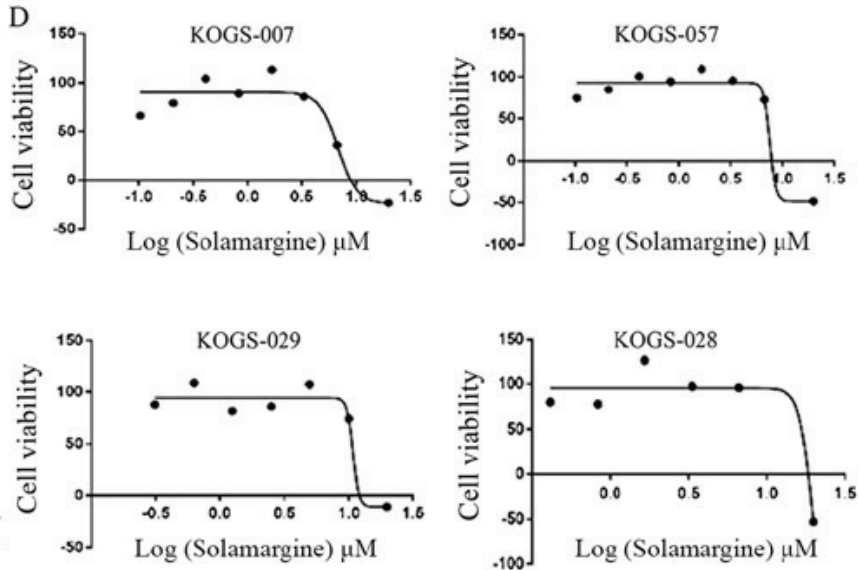

E

\begin{tabular}{|c|c|c|}
\hline Cell ID & \multicolumn{2}{|c|}{ Solamargine } \\
\hline Cell line & $\mathrm{IC}_{50}(\mu \mathrm{M})$ & Hillslope \\
\hline KOGS-029 & 18.80 & -18.95 \\
\hline KOGS-007 & 7.50 & -15.04 \\
\hline KOGS-057 & 6.81 & -5.02 \\
\hline KOGS-028 & 19.78 & -10.21 \\
\hline
\end{tabular}

Figure 6. Effect of Solamargine in a xenograft mouse model and Solamargine sensitivity $\left(\mathrm{IC}_{50}\right)$ in primary GC cells from patients. (A) Mice were sacrificed and the tumors were collected. Tumor growth was significantly inhibited following treatment with Solamargine. ${ }^{\text {"P }}<0.05$ vs. control. (B) Early apoptosis cells were detected by TUNEL staining. Magnification, x63. ${ }^{*} \mathrm{P}<0.05$ vs. control. (C) H\&E staining was used to analyze the necrotic area (\%) of the GC xenograft. Upper panel, magnification, $\mathrm{x} 5$; bottom panel, magnification, $\mathrm{x} 20$. "P<0.05 vs. control. (D) Primary cells from four patients who underwent radical gastrectomy were treated with appropriate doses of Solamargine for $72 \mathrm{~h}$. (E) Solamargine sensitivity $\left(\mathrm{IC}_{50}\right)$ in primary $\mathrm{GC}$ cells from four patients. GC, gastric cancer; IC50, half-maximal inhibitory concentration (48 h); TUNEL, terminal deoxynucleotidyl transferase-mediated dUTP-biotin nick end labeling.

reported lncPINT and lncNEAT1_2 to be closely associated with MAPKs $(29,30)$. Based on the findings of the present study that Solamargine inhibited the phosphorylation of MAPKs, whether the expression of lncPINT and lncNEAT1_2 may be affected was investigated. The results demonstrated that the expression levels of lncPINT and lncNEAT1_2 were significantly increased in the Solamargine-treated group compared with in the control group of SGC7901 cells $(5 \mu \mathrm{M}$, $\mathrm{P}<0.05 ; 10 \mu \mathrm{M}, \mathrm{P}<0.01$; Fig. $4 \mathrm{C}$ and $\mathrm{D})$. Additionally, the upregulated expression levels appeared to be dose-dependent; however, treatment with $5 \mu \mathrm{M}$ Solamargine revealed a non-significant increase in the expression of lncNEAT1_2 in BGC823 cells. According to the findings of the present study, Solamargine inhibited the phosphorylation of MAPKs and upregulated the expression of IncPINT and IncNEAT1_2 in a dose-dependent manner.

Inhibition of Erk1/2 MAPK increases the expression of lncNEAT1_2. The present study demonstrated that Solamargine reduced the phosphorylation of Erk1/2 MAPK and increased the expression of IncNEAT1_2. LncPINT and one splice variant of lncNEAT1 were reported to be regulated in an Erk-dependent manner (20). To understand the potential mechanism by which Solamargine affects GC, the interactions between Erk1/2 MAPK and lncNEAT1_2 were investigated. The results revealed that treatment with an exogenous inhibitor of Erk1/2 MAPK (U0126) reduced the expression of Erk1/2 MAPK and significantly enhanced the expression of lncNEAT1_2 in a time-dependent manner (Fig. 5A-C), which was consistent with the effects of Solamargine. Knockdown of lncNEAT1_2
(Fig. 5D-G) had no notable effect on the expression of Erk1/2 MAPK or its phosphorylation. Collectively, the results suggested that Erk1/2 MAPK is upstream of lncNEAT1_2 and that Solamargine increased the expression of lncNEAT1_2 via the inhibition of Erk1/2 MAPK signaling.

Knockdown of IncNEAT1_2 attenuates the inhibitory effects of Solamargine on GC cells. The aforementioned findings indicated that Solamargine regulated the expression of lncPINT and lncNEAT1_2. Furthermore, to confirm the observed inhibition, the effects of Solamargine on GC cell viability were investigated by downregulating the expression of lncNEAT1_2 in GC cells; the IncuCyte ZOOM Live-Cell Analysis System was employed. The results demonstrated that knockdown of lncNEAT1_2 significantly attenuated the inhibitory effects of Solamargine on GC cells (Fig. 5H). The results suggested that IncNEAT1_2 is a potentially important therapeutic target for Solamargine.

In vivo antitumor activity of Solamargine. To further assess the activity of Solamargine in vivo, alterations in tumor growth in response to Solamargine were investigated within a tumor xenograft model. As presented in Fig. 6A, the tumor volume in the Solamargine group was significantly reduced compared with in the control group. Based on the present study, Solamargine served a crucial role in promoting the apoptosis in GC cells. A TUNEL assay and H\&E staining were performed using excised tumor sections. The results revealed a significant increase in the number of early apoptotic cells in the Solamargine group (Fig. 6B). Furthermore, the 
results of $H \& E$ staining suggested that the necrotic area in the Solamargine group was significantly increased compared with in the control (Fig. 6C). In summary, the results indicated that Solamargine exhibited an antitumor effect and promoted the apoptosis of GC in vivo.

Solamargine sensitivity of primary GC cells from patients. The cell lines employed in the present study exhibited cancer-associated characteristics without the heterogeneity observed in primary tumors (31). To study the responsiveness of patients to Solamargine treatment, cell viability assays were performed on primary GC cells. Primary cells from four patients who underwent radical gastrectomy were treated with appropriate amounts of Solamargine for over $72 \mathrm{~h}$. A drug sensitivity assay was performed and Solamargine was observed to suppress the viability of GC cells from all four patients (Fig. 6D); the $\mathrm{IC}_{50}$ values were determined (Fig. 6E). The result of the present study indicated that the effects of Solamargine on primary GC cells obtained from patients were similar to that of GC cell lines.

\section{Discussion}

A previous study reported that Solamargine exhibited an inhibitory effect on a variety of tumor cell lines; however, the role of Solamargine in GC cells requires further investigation (9,32-34). Studies of Solamargine with in other cancer types demonstrated variations in the $\mathrm{IC}_{50}$ values from 0.91 to $26.66 \mu \mathrm{M}$ (32). The present study observed a significant inhibitory effect on GC in response to $10 \mu \mathrm{M}$ Solamargine by using the IncuCyte ZOOM Live-Cell system. Based on the aforementioned analysis and results, concentrations of $0-10 \mu \mathrm{M}$ Solamargine were applied in the present study. To the best of our knowledge, the present study is the first to report that Solamargine induced the apoptosis and suppressed the viability of GC cell lines.

A few molecular pathways can influence the anti-tumor activity of Solamargine, including the tumor necrosis factor receptor (35), B-cell lymphoma 2 (Bcl-2)/Bcl-2-associated X (36) and MAPK signaling pathways $(16,33)$. MAPKs are a widely conserved family of serine/threonine protein kinases (37). Previously, studies have reported numerous biological functions of cells to be regulated by the MAPK signaling pathway, including proliferation, apoptosis and metastasis (15,38-42). In addition, its activation was closely associated with carcinogenesis and tumor development (43). Targeting the members of the MAPK family was therefore a prospective approach to the management of incurable GC. The present study demonstrated that Solamargine inhibited GC by suppressing the phosphorylation of Erk1/2, one of the key members of the MAPK family, in a dose-dependent manner. The results of the present study suggested that the MAPK network is a crucial signaling pathway involved in the inhibitory effects of Solamargine on GC.

LncNEAT1 is expressed as two splicing variants that differ in length, namely, NEAT1_1 and NEAT1_2 (23). A previous study reported that the two splicing variants exhibited differing effects on tumor progression (44). Interestingly, by using the murine hippocampus as a model, Blüthgen et al (45) revealed that the induction of lncPINT and the induction of one splicing variant of lncNEAT1 were Erk-dependent $(46,47)$. MAPK is an important signaling pathway within living organisms in response to extracellular stimuli (31). The present study proposed that lncPINT and lncNEAT1 were Erk-dependent in GC as reported in murine hippocampus (46). Based on the reports that Solamargine inhibited the MAPK pathway, the roles of lncPINT and lncNEAT1_2 were investigated in the present study to determine the potential mechanism underlying the inhibitory effects of Solamargine. The results demonstrated that Solamargine inhibited the phosphorylation of Erk1/2 MAPK in GC and increased the expression of lncPINT and lncNEAT1_2. In addition, downregulating the expression of lncNEAT1_2 significantly reduced the inhibitory effects of Solamargine on GC. To the best of our knowledge, the present study is the first to reveal that Solamargine suppressed the viability of GC cells mainly via the overexpression of lncPINT and lncNEAT1_2. Thus, a novel strategy for investigating the mechanism of Solamargine and insight into clinical anticancer treatment against GC were reported; however to understand the mechanism of this regulatory axis, further investigation is required.

At present, based on the scientific evidence available, Solamargine may be considered as a promising treatment against cancer; however, its anticancer potential in vivo and within patients remains unclear $(11,12,48)$. In the present study, the effects of Solamargine on GC were also observed in vivo via nude mice and in primary cells from patients. The present study reported that experiments in vivo and in primary cells from patients also suggested that Solamargine exerted an inhibitory effect on tumor growth in GC. In future studies, other modes of administration with increased efficiency, such as intravenous administration, may also be studied. In addition, patient-derived tumor xenografts can be employed to verify the effects of Solamargine, and ultimately, clinical trials may be performed to assess its efficacy.

Collectively, the results of the present study suggest that Solamargine exerted an inhibitory effect on GC and that this effect was achieved by inducing the expression of lncPINT and lncNEAT1_2 via the inhibition of Erk1/2 MAPK phosphorylation. Therefore, Solamargine may be considered as a potential therapeutic agent for the treatment of $\mathrm{GC}$ and may be applied in future clinical studies.

\section{Acknowledgements}

Not applicable.

\section{Funding}

This study was supported by the 'San Ming' Project (grant no. SZSM201612051, Shenzhen, China).

\section{Availability of data and materials}

The analyzed data sets generated during the study are available from the corresponding authors on reasonable request.

\section{Authors' contributions}

JJ contributed to the design of the study. LiaZ and GL wrote the manuscript. RF performed the experiments. XW analyzed the 
data. YH, HD, BD, SA, LiZ and ZS provided the Solamargine and technical assistance. All authors read and approved the final manuscript.

\section{Ethics approval and consent to participate}

The collection of primary GC cells from patients who underwent radical gastrectomy for the present study was approved by the Ethics Committee of Peking University Cancer Hospital and Institute (Beijing, China). Informed consent was provided by all patients. The animal study was approved by Peking University Cancer Hospital Research Ethics Committee (Beijing, China) and was conducted in strict accordance with the Guidelines for the Care and Use of Laboratory Animals of the Peking University Cancer Hospital and Institute (26).

\section{Patient consent for publication}

Not applicable.

\section{Competing interests}

ZS has ownership interest (including patents) in K2 Oncology.

\section{References}

1. Torre LA, Bray F, Siegel RL, Ferlay J, Lortet-Tieulent J and Jemal A: Global cancer statistics, 2012. CA Cancer J Clin 65: 87-108, 2015

2. Siegel RL, Miller KD and Jemal A: Cancer Statistics, 2017. CA Cancer J Clin 67: 7-30, 2017.

3. Xi J, Feng J, Li Q, Li X and Zeng S: The long non-coding RNA lncFOXO1 suppresses growth of human breast cancer cells through association with BAP1. Int J Oncol 50: 1663-1670, 2017.

4. Xu Y, Zheng Y, Liu H and Li T: Modulation of IGF2BP1 by long non-coding RNA HCG11 suppresses apoptosis of hepatocellular carcinoma cells via MAPK signaling transduction. Int J Oncol 51: 791-800, 2017.

5. Zhang $\mathrm{R}$ and Xia T: Long non-coding RNA XIST regulates PDCD4 expression by interacting with miR-21-5p and inhibits osteosarcoma cell growth and metastasis. Int J Oncol 51: $1460-1470,2017$

6. Burger T, Mokoka T, Fouché G, Steenkamp P, Steenkamp V and Cordier W: Solamargine, a bioactive steroidal alkaloid isolated from Solanum aculeastrum induces non-selective cytotoxicity and P-glycoprotein inhibition. BMC Complement Altern Med 18: $137,2018$.

7. Marín-Béjar O, Mas AM, González J, Martinez D, Athie A, Morales X, Galduroz M, Raimondi I, Grossi E, Guo S, et al: The human lncRNA LINC-PINT inhibits tumor cell invasion through a highly conserved sequence element. Genome Biol 18: 202, 2017.

8. Chen Y, Tang Q, Wu J, Zheng F, Yang L and Hann SS: Inactivation of PI3-K/Akt and reduction of SP1 and p65 expression increase the effect of solamargine on suppressing EP4 expression in human lung cancer cells. J Exp Clin Cancer Res 34: 154, 2015

9. Ding X, Zhu FS, Li M and Gao SG: Induction of apoptosis in human hepatoma SMMC-7721 cells by solamargine from Solanum nigrum L. J Ethnopharmacol 139: 599-604, 2012.

10. Sun L, Zhao Y, Li X, Yuan H, Cheng A and Lou H: A lysosomal-mitochondrial death pathway is induced by solamargine in human K562 leukemia cells. Toxicol In Vitro 24 $1504-1511,2010$

11. Xie X, Zhu H, Zhang J, Wang M, Zhu L, Guo Z, Shen W and Wang D: Solamargine inhibits the migration and invasion of HepG2 cells by blocking epithelial-to-mesenchymal transition. Oncol Lett 14: 447-452, 2017.

12. Shiu LY, Chang LC, Liang CH, Huang YS, Sheu HM and Kuo KW: Solamargine induces apoptosis and sensitizes breast cancer cells to cisplatin. Food Chem Toxicol 45: 2155-2164, 2007.
13. Xiang S, Zhang Q, Tang Q, Zheng F, Wu J, Yang L and Hann SS: Activation of AMPK $\alpha$ mediates additive effects of solamargine and metformin on suppressing MUC1 expression in castration-resistant prostate cancer cells. Sci Rep 6: 36721, 2016.

14. Wu YH, Chiu WT, Young MJ, Chang TH, Huang YF and Chou CY: Solanum Incanum Extract Downregulates Aldehyde Dehydrogenase 1-Mediated Stemness and Inhibits Tumor Formation in Ovarian Cancer Cells. J Cancer 6: 1011-1019, 2015.

15. Cui CZ, Wen XS, Cui M, Gao J, Sun B and Lou HX: Synthesis of solasodine glycoside derivatives and evaluation of their cytotoxic effects on human cancer cells. Drug Discov Ther 6: 9-17, 2012.

16. Zhou Y, Tang Q, Zhao S, Zhang F, Li L, Wu W, Wang Z and Hann S: Targeting signal transducer and activator of transcription 3 contributes to the solamargine-inhibited growth and -induced apoptosis of human lung cancer cells. Tumour Biol 35: 8169-8178, 2014.

17. Chen Y, Tang Q, Xiao Q, Yang L and Hann SS: Targeting EP4 downstream c-Jun through ERK1/2-mediated reduction of DNMT1 reveals novel mechanism of solamargine-inhibited growth of lung cancer cells. J Cell Mol Med 21: 222-233, 2017.

18. Schmitt AM and Chang HY: Long Noncoding RNAs in Cancer Pathways. Cancer Cell 29: 452-463, 2016.

19. Li L, Zhang GQ, Chen H, Zhao ZJ, Chen HZ, Liu H, Wang G, Jia YH, Pan SH, Kong R, et al: Plasma and tumor levels of Linc-pint are diagnostic and prognostic biomarkers for pancreatic cancer. Oncotarget 7: 71773-71781, 2016.

20. Yang X, Qu S, Wang L, Zhang H, Yang Z, Wang J, Dai B, Tao K, Shang R, Liu Z, et al: PTBP3 splicing factor promotes hepatocellular carcinoma by destroying the splicing balance of NEAT1 and pre-miR-612. Oncogene 37: 6399-6413, 2018.

21. Zhang H, Cai Y, Zheng L, Zhang Z, Lin X and Jiang N: Long noncoding RNA NEAT1 regulate papillary thyroid cancer progression by modulating miR-129-5p/KLK7 expression. J Cell Physiol 233: 6638-6648, 2018.

22. Yu B and Shan G: Functions of long noncoding RNAs in the nucleus. Nucleus 7: 155-166, 2016.

23. Yu X, Li Z, Zheng H, Chan MT and Wu WK: NEAT1: A novel cancer-related long non-coding RNA. Cell Prolif 50: 50, 2017.

24. Mello SS, Sinow C, Raj N, Mazur PK, Bieging-Rolett K, Broz DK, Imam JFC, Vogel H, Wood LD, Sage J, et al: Neat1 is a p53-inducible lincRNA essential for transformation suppression. Genes Dev 31: 1095-1108, 2017.

25. Livak KJ and Schmittgen TD: Analysis of relative gene expression data using real-time quantitative PCR and the 2(-Delta Delta C(T)) method. Methods 25: 402-408, 2001

26. National Research Council (US) Committee for the Update of the Guide for the Care and Use of Laboratory Animals: Guide for the Care and Use of Laboratory Animals. 8th edition. National Academies Press (US), Washington, DC, 2011.

27. Julien $\mathrm{O}$ and Wells JA: Caspases and their substrates. Cell Death Differ 24: 1380-1389, 2017.

28. Choi EO, Park C, Hwang HJ, Hong SH, Kim GY, Cho EJ, Kim WJ and Choi YH: Baicalein induces apoptosis via ROS-dependent activation of caspases in human bladder cancer 5637 cells. Int J Oncol 49: 1009-1018, 2016.

29. Pedersen AE, Bregenholt S, Johansen B, Skov S and Claesson MH: MHC-I-induced apoptosis in human B-lymphoma cells is dependent on protein tyrosine and serine/threonine kinases. Exp Cell Res 251: 128-134, 1999.

30. Cross TG, Scheel-Toellner D, Henriquez NV, Deacon E, Salmon M and Lord JM: Serine/threonine protein kinases and apoptosis. Exp Cell Res 256: 34-41, 2000.

31. Correa BRS, Hu J, Penalva LOF, Schlegel R, Rimm DL, Galante PAF and Agarwal S: Patient-derived conditionally reprogrammed cells maintain intra-tumor genetic heterogeneity. Sci Rep 8: 4097, 2018.

32. Kalalinia F and Karimi-Sani I: Anticancer Properties of Solamargine: A Systematic Review. Phytother Res 31: 858-870, 2017.

33. Al Sinani SS, Eltayeb EA, Coomber BL and Adham SA: Solamargine triggers cellular necrosis selectively in different types of human melanoma cancer cells through extrinsic lysosomal mitochondrial death pathway. Cancer Cell Int 16: 11, 2016.

34. Del Bufalo D, Biroccio A, Trisciuoglio D, Bruno T, Floridi A, Aquino A and Zupi G: Bcl-2 has differing effects on the sensitivity of breast cancer cells depending on the antineoplastic drug used. Eur J Cancer 38: 2455-2462, 2002. 
35. Hsu SH, Tsai TR, Lin CN, Yen MH and Kuo KW: Solamargine purified from Solanum incanum Chinese herb triggers gene expression of human TNFR I which may lead to cell apoptosis. Biochem Biophys Res Commun 229: 1-5, 1996.

36. Xie X, Zhu H, Yang H, Huang W, Wu Y, Wang Y, Luo Y, Wang D and Shao G: Solamargine triggers hepatoma cell death through apoptosis. Oncol Lett 10: 168-174, 2015.

37. Morrison DK and Davis RJ: Regulation of MAP kinase signaling modules by scaffold proteins in mammals. Annu Rev Cell Dev Biol 19: 91-118, 2003.

38. Kyriakis JM and Avruch J: Mammalian MAPK signal transduction pathways activated by stress and inflammation: A 10-year update. Physiol Rev 92: 689-737, 2012.

39. Yoda Y, Takeshima H, Niwa T, Kim JG, Ando T, Kushima R, Sugiyama T, Katai $H$, Noshiro $H$ and Ushijima T: Integrated analysis of cancer-related pathways affected by genetic and epigenetic alterations in gastric cancer. Gastric Cancer 18: 65-76, 2015.

40. Xie J, Jin B, Li DW, Shen B, Cong N, Zhang TZ and Dong P: ABCG2 regulated by MAPK pathways is associated with cancer progression in laryngeal squamous cell carcinoma. Am J Cancer Res 4: 698-709, 2014.

41. Deng J, Qian Y, Geng L, Xie H, Wang Y, Jiang G, Zhou L, Zhang $M$ and Zheng S: Involvement of ERK and JNK pathways in IFN- $\gamma$-induced B7-DC expression on tumor cells. J Cancer Res Clin Oncol 137: 243-250, 2011.

42. Wang Z, Wang W, Xu S, Wang S, Tu Y, Xiong Y, Mei J and Wang C: The role of MAPK signaling pathway in the Her-2-positive meningiomas. Oncol Rep 36: 685-695, 2016.
43. Peluso I, Yarla NS, Ambra R, Pastore G and Perry G: MAPK signalling pathway in cancers: Olive products as cancer preventive and therapeutic agents. Semin Cancer Biol: Sep 11, 2017 (Epub ahead of print).

44. Wu Y, Yang L, Zhao J, Li C, Nie J, Liu F, Zhuo C, Zheng Y, Li B, Wang Z, et al: Nuclear-enriched abundant transcript 1 as a diagnostic and prognostic biomarker in colorectal cancer. Mol Cancer 14: 191, 2015.

45. Blüthgen N, van Bentum M, Merz B, Kuhl D and Hermey G: Profiling the MAPK/ERK dependent and independent activity regulated transcriptional programs in the murine hippocampus in vivo. Sci Rep 7: 45101, 2017.

46. Csibi A, Fendt SM, Li C, Poulogiannis G, Choo AY, Chapski DJ, Jeong SM, Dempsey JM, Parkhitko A, Morrison T, et al: The mTORC1 pathway stimulates glutamine metabolism and cell proliferation by repressing SIRT4. Cell 153: 840-854, 2013.

47. Shukla V, Coumoul X, Wang RH, Kim HS and Deng CX: RNA interference and inhibition of MEK-ERK signaling prevent abnormal skeletal phenotypes in a mouse model of craniosynostosis. Nat Genet 39: 1145-1150, 2007.

48. Nishie K, Norred WP and Swain AP: Pharmacology and toxicology of chaconine and tomatine. Res Commun Chem Pathol Pharmacol 12: 657-668, 1975.

(i) $\Theta$ This work is licensed under a Creative Commons Attribution-NonCommercial-NoDerivatives 4.0 International (CC BY-NC-ND 4.0) License. 